\title{
Expression of fatty acid binding protein 4 is involved in the cell growth of oral squamous cell carcinoma
}

\author{
DOKSA LEE ${ }^{1}$, KOICHIRO WADA $^{2}$, YOSHITAKA TANIGUCHI ${ }^{1}$, HANI AL-SHAREEF $^{1}$, TOMOTAKE MASUDA ${ }^{1}$, \\ YU USAMI $^{3}$, TOMONAO AIKAWA ${ }^{1}$, MASAYA OKURA ${ }^{1}$, YOSHINORI KAMISAKI $^{2}$ and MIKIHIKO KOGO ${ }^{1}$ \\ ${ }^{1}$ First Department of Oral and Maxillofacial Surgery and ${ }^{2}$ Department of Pharmacology, Graduate School of Dentistry, \\ Osaka University; ${ }^{3}$ Clinical Laboratory, Osaka University Dental Hospital, Suita, Osaka 565-0871, Japan
}

Received November 30, 2013; Accepted December 27, 2013

DOI: $10.3892 /$ or.2014.2975

\begin{abstract}
Fatty acid binding proteins (FABPs) are a family of small and highly conserved lipid chaperone molecules with highly varied functions. Among them, fatty acid binding protein 4 (FABP4, also known as aP2) is highly expressed by adipocytes, macrophages and dendritic cells. Although the role of FABP4 in cancer is still unclear, it has been reported to be highly expressed by human tumors such as ovarian and bladder cancers. In the present study, we investigated the expression and role of FABP4 in oral squamous cell carcinoma (SCC) and its expression in oral SCC tissues. Immunohistochemical staining revealed that $\mathrm{FABP} 4$ expression in the tumor tissue was much higher than that in the non-tumor area of the same specimen. In the in vitro studies, an FABP4-knockdown SCC cell line (established through FABP4-specific siRNA) showed inhibited growth, and inhibited expression and activation of mitogen-activated protein kinase (MAPK). These results indicate that expression of FABP4 plays an important role in the cell growth of oral SCC through the MAPK pathway.
\end{abstract}

\section{Introduction}

Oral squamous cell carcinoma (SCC) is a major neoplasm of the oral cavity with an increasing rate of incidence (1-3). The optimal therapy for early oral SCC is surgery, but the overall survival rate has exhibited only a slight change (1-3). Therefore, more effective therapies for oral SCC are needed.

Fatty acid binding proteins (FABPs) are a family of small and highly conserved lipid chaperone molecules that bind long-chain fatty acids and other hydrophobic ligands. Their functions are wide ranging (4-6). Among them, fatty acid

Correspondence to: Dr Doksa Lee, First Department of Oral and Maxillofacial Surgery, Graduate School of Dentistry, Osaka University, 1-8 Yamadaoka, Suita, Osaka 565-0871, Japan

E-mail: doksa.lee@gmail.com

Key words: fatty acid binding protein 4, squamous cell carcinoma, oral cancer, tongue cancer, cellular proliferation, mitogen-activated protein kinase binding protein 4 (FABP4, also known as aP2) is highly expressed in adipocytes, macrophages and dendritic cells $(5,7)$. As a result of its distribution, FABP4 is the most extensively researched FABP in endocrinology and metabolomics. FABP4 affects metabolic syndrome progression; FABP4-deficient mice were found to have reduced hyperinsulinemia and insulin resistance in obesity $(7,8)$ and showed protection from atherosclerosis (9).

However, little is known concerning the role of FABP4 in cancer, including oral SCC. Recently, Nieman et al (10) reported that adipocytes promote ovarian cancer metastasis and tumor cell growth by providing energy mediated by FABP4. Therefore, increased FABP4 expression may affect the growth of various tumor types. Our research group also reported that molecules controlled by peroxisome proliferator-activated receptor $\gamma(\operatorname{PPAR} \gamma)$ play key roles in SCC growth (11-15). As FABP4 is known to mediate transcription with PPAR $\gamma(4,16)$, we hypothesized that FABP4 may regulate SCC growth. Therefore, in the present study, we investigated FABP4 expression and its effects on SCC of the tongue.

\section{Materials and methods}

Tissue samples. All clinical studies were approved by the Ethics Committee of Osaka University Dental Hospital, Osaka. Twenty-seven SCC specimens from resected tongue tissue were obtained at the Osaka University Dental Hospital during 1986-2008 after patient informed consent (Table I). Patients received no preoperative therapy, including chemotherapy and irradiation therapy. The age range of the patients was 30-92 years $(61.6 \pm 16.4$ years, mean age \pm SD); 17 patients were men and 10 were women.

Antibodies. The anti-FABP4 polyclonal antibody was obtained from Bioss Inc. (Woburn, MA, USA). Antibodies against p44/42MAPK and the phosphorylated p44/42MAPK antibody were from Cell Signaling Technology (Beverly, MA, USA).

Immunohistochemical staining and evaluation of FABP4 expression. FABP4 expression in tissues was detected by an anti-FABP4 antibody using standard immunohistochemical techniques (12-15). Formalin-fixed and paraffin-embedded continuous sections were selected and sliced into $5-\mu \mathrm{m}$ 
Table I. Characteristics of the patients with squamous cell carcinoma of the tongue, their histological diagnosis and expression of FABP4 in the tissue specimens.

FABP4 expression

\begin{tabular}{|c|c|c|c|c|c|}
\hline Case no. & Age (years) & Gender & Differentiation & Tumor area & Non-tumor area \\
\hline 1 & 34 & Male & Well-differentiated SCC & 1 & 0 \\
\hline 2 & 46 & Female & Well-differentiated SCC & 2 & 0 \\
\hline 3 & 57 & Male & Well-differentiated SCC & 1 & 1 \\
\hline 4 & 64 & Male & Well-differentiated SCC & 0 & 0 \\
\hline 5 & 69 & Male & Well-differentiated SCC & 0 & 0 \\
\hline 6 & 71 & Female & Well-differentiated SCC & 1 & 0 \\
\hline 7 & 71 & Male & Well-differentiated SCC & 1 & 0 \\
\hline 8 & 72 & Male & Well-differentiated SCC & 2 & 0 \\
\hline 9 & 78 & Female & Well-differentiated SCC & 1 & 0 \\
\hline 10 & 79 & Male & Well-differentiated SCC & 1 & 1 \\
\hline 11 & 47 & Male & Moderately differentiated SCC & 1 & 0 \\
\hline 12 & 61 & Male & Moderately differentiated SCC & 1 & 0 \\
\hline 13 & 61 & Female & Moderately differentiated SCC & 1 & 0 \\
\hline 14 & 72 & Male & Moderately differentiated SCC & 1 & 0 \\
\hline 15 & 77 & Male & Moderately differentiated SCC & 1 & 2 \\
\hline 16 & 83 & Female & Moderately differentiated SCC & 3 & 2 \\
\hline 17 & 92 & Female & Moderately differentiated SCC & 1 & 0 \\
\hline 18 & 30 & Male & Poorly differentiated SCC & 2 & 1 \\
\hline 19 & 33 & Male & Poorly differentiated SCC & 0 & 0 \\
\hline 20 & 38 & Male & Poorly differentiated SCC & 0 & 0 \\
\hline 21 & 51 & Female & Poorly differentiated SCC & 0 & 0 \\
\hline 22 & 52 & Male & Poorly differentiated SCC & 2 & 0 \\
\hline 23 & 55 & Female & Poorly differentiated SCC & 2 & 0 \\
\hline 24 & 65 & Male & Poorly differentiated SCC & 1 & 3 \\
\hline 25 & 67 & Male & Poorly differentiated SCC & 3 & 2 \\
\hline 26 & 41 & Female & Differentiation unknown SCC & 0 & 0 \\
\hline 27 & 68 & Female & Differentiation unknown SCC & 2 & 0 \\
\hline
\end{tabular}

Expression of FABP4 by immunohistochemical staining in the tumor and non-tumor areas is scored and expressed as 0-3.

sections. Briefly, incubation with an anti-FABP4 polyclonal antibody was performed at $4^{\circ} \mathrm{C}$ for $16 \mathrm{~h}$; sections were then washed. After applying the secondary antibody, the Vectastain ABC kit (Vector Laboratories, Burlingame, CA, USA) was used with a 3,3'-diaminobenzidine substrate kit, according to the manufacturer's instructions. The staining endpoint was determined when the standard tissue sections were constantly stained at the intensity as previously described $(12,17)$.

The intensity of the immunohistochemical staining with the anti-FABP4 antibody was evaluated by scoring according to four groups: $0,<10 \% ; 1,10-20 \% ; 2,>20-50 \%$; and $3,>50 \%$ of the cells exhibiting cytoplasmic staining $(12,17)$. To confirm the reproducibility, the anti-FABP4 immunohistochemical staining was re-evaluated by a pathologist who was unaware of the original assessment. Non-tumor areas were selected as comparatively normal areas separated from the tumor areas by an appropriate distance and confirmed by the pathologist $(14,15)$.
Cell culture and cell growth assay. We used a human oral SCC cell line (SAS) that was established from tongue SCC (13). Cells were maintained in DMEM containing $10 \%$ fetal bovine serum (FBS) at $37^{\circ} \mathrm{C}$ under $0.5 \% \mathrm{CO}_{2}$. For the cell growth experiment, cells were trypsinized and replated onto culture dishes $(11-15,17,18)$. SCC cells were counted using a Countess Automated Cell Counter (Invitrogen, Eugene, OR, USA). Inhibition of cell growth was compared with the vehicletreated controls.

RNA interference approach. The SAS cells were trypsinized and resuspended in DMEM without FBS, and then separated placing $\sim 2 \times 10^{5}$ cells in each dish. The FABP4-specific siRNA (Stealth siRNA) was purchased from Invitrogen Japan (Tokyo, Japan). We purchased three sequences and performed preparatory experiments to determine the most effective sequence. The sequences of the selected FABP4-siRNA were: sense, 5'-CAC CAUUAAAUCUGAAAGUACCUUU-3' and antisense, 


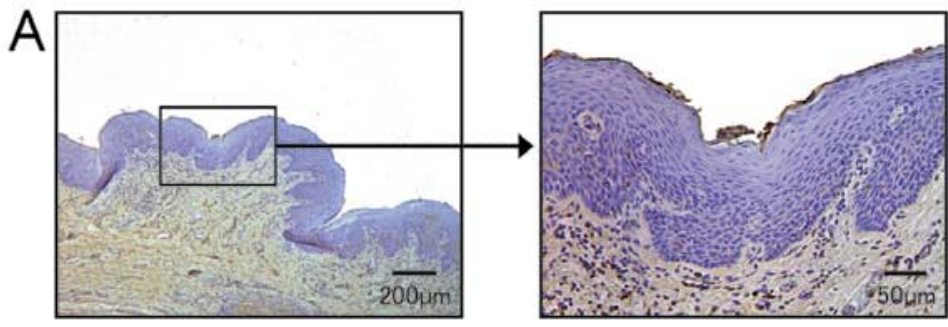

Non-tumor area

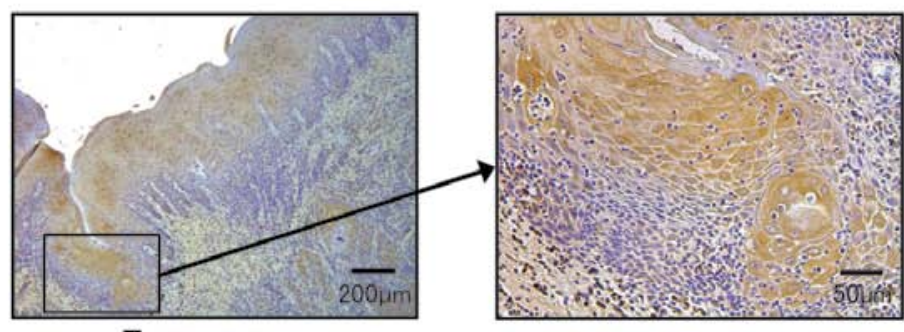

Tumor area

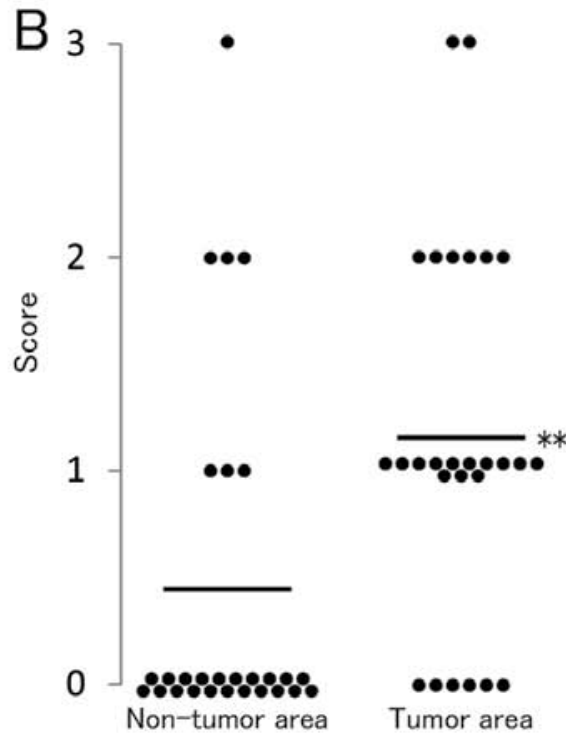

Tumor area

Figure 1. Expression of fatty acid binding protein 4 (FABP4) in tumor cells in tongue squamous cell carcinoma. (A) FABP4 expression in the tumor area (upper panel) of primary tongue squamous cell carcinoma and thevnon-tumor area (lower panel) from the same tissue section (case 13 in Table I) using immunohistochemical staining. The brown color shows positive FABP4 staining; blue is a counterstain. (B) Comparison of FABP4 expression between tumor and non-tumor areas. The score of the distribution of FABP4 expression is indicated by black circles from the data in Table I. A horizontal line shows the average score. Data indicate the mean \pm SEM from 27 cases. ${ }^{* * *} \mathrm{P}<0.01$.

\section{5'-AAAGGUACUUUCAGAUUUAAUGGUG-3'. For transfec-} tion, FABP4-siRNA or a negative control (Stealth RNAi negative control duplex; Invitrogen Japan) solution was added to the DMEM containing Lipofectamine RNAiMax (Invitrogen Japan) and incubated for $20 \mathrm{~min}$ at room temperature to create the transfection mixture. The transfection mixture was then added to the cells at the indicated final siRNA concentrations. Following $24 \mathrm{~h}$ of transfection, the medium was replaced by DMEM containing $10 \%$ FBS, at which time viable cells were counted using a Countess Automated Cell Counter. Cell growth was expressed as a percentage of the vehicle-treated control growth.

Western blot analysis. Adherent or suspended cells were washed in PBS, and the cell extracts were prepared by lysing the cells in lysis buffer. Proteins were separated by electrophoresis using $10 \%$ SDS-PAGE and transferred to a nitrocellulose membrane (Millipore, Bedford, MA, USA). Detection of proteins was performed with each polyclonal antibody and visualized using an ECL detection kit (Amersham, London, UK) following the manufacturer's recommended procedure.

Statistical analysis. Results are expressed as the means \pm SEM or \pm SD. Statistical comparisons were carried out using the Student's t-test or the Scheffé's method after analysis of variance. $\mathrm{P}<0.05$ was considered to indicate a statistically significant result.

\section{Results}

Tongue SCC tissues express FABP4. We stained tongue SCC tissues using the FABP4-specific antibody. Within single tumor specimens, the non-tumor areas were unstained (Fig. 1A, upper panel), whereas tumor areas showed positive FABP4 staining (Fig. 1A, lower panel). According to the scoring as described above, FABP4 expression between the non-tumor and the tumor area differed significantly (Fig. 1B), and FABP4 was expressed in the tumor areas, but not in the normal tissues.

FABP4-specific siRNA suppresses the growth of tongue SCC. Treatment with FABP4-siRNA markedly decreased FABP4 protein levels in the SAS cells (Fig. 2A), and suppressed SAS cell growth in a concentration-dependent manner (Fig. 2B). Inhibition of SCC growth was also visibly altered (Fig. 2C), and significantly differed between the SAS controls and the FABP4-knockdown SAS cells (Fig. 2D).

FABP4-specific siRNA inhibits expression and phosphorylation of mitogen-activated protein kinase (MAPK). To investigate the mechanisms involved in the growth inhibition induced by suppression of FABP4, we analyzed a type of MAPK, serine/threonine protein kinases. Since they affect cell proliferation, survival and differentiation, aberrant MAPK cascades contribute to cancer and other diseases (19-21). Therefore, we studied the effects of FABP4 knockdown on MAPK expression and phosphorylation. Western blot analysis showed decreased phosphorylated MAPK (pMAPK; Fig. 3, middle panel). Notably, MAPK expression itself was also regulated by $F A B P 4$ knockdown (Fig. 3, upper panel).

\section{Discussion}

FABP4 expression has been reported in various types of tumors such as ovarian and bladder cancers $(10,22)$, and FABP5 (E-FAPB) has been found in oral SCC $(23,24)$. However, the expression and exact role of FABP4 in oral SCC have not been widely investigated

In the present study, using an immunohistochemical approach, we consistently found significantly higher expression of FABP4 protein in the tumor area of tongue SCC than 

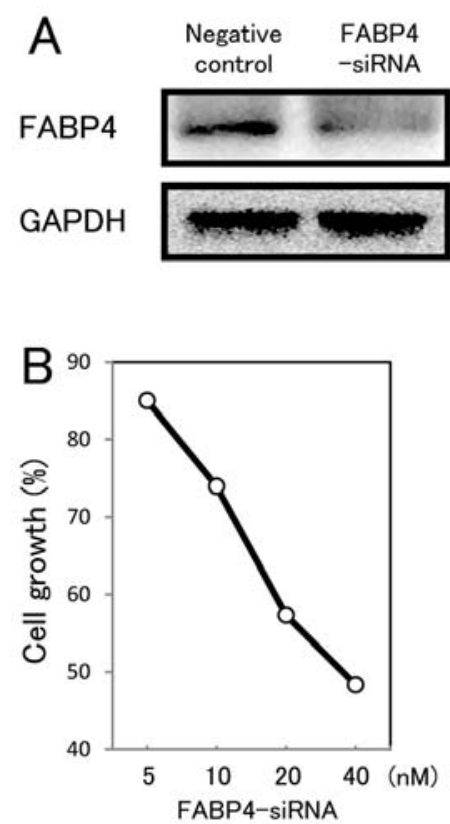

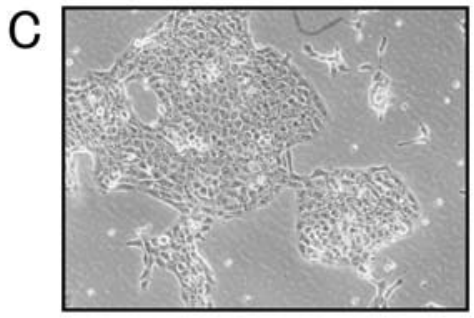

Negative control

$(40 \mathrm{nM})$

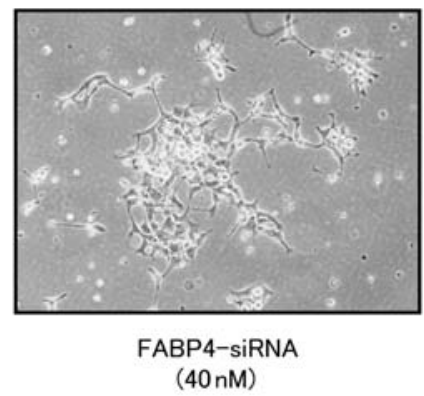

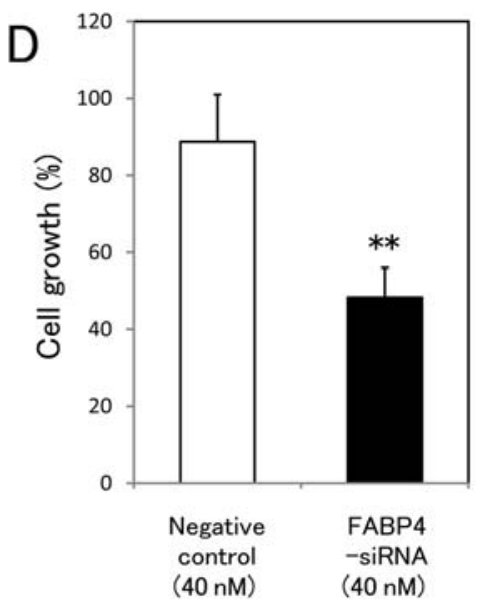

Figure 2. Effect of FABP4 knockdown on the growth of SCC cells treated with FABP4-siRNA. (A) Western blot analysis of FABP4 expression in SAS cells treated with $F A B P 4$-specific siRNA $(40 \mathrm{nM})$ or negative control siRNA $(40 \mathrm{nM})$. Samples were collected $12 \mathrm{~h}$ after treatment. $G A P D H$ was used as the standard for equivalent application. (B) A concentration-dependent effect of $F A B P 4$-specific siRNA on cell growth. SAS cells were treated with $F A B P 4$ specific siRNA for $24 \mathrm{~h}$, followed by additional culture for $72 \mathrm{~h}$. Left axis presents the percentage of cell growth compared with vehicle (non-siRNA) control. (C) Typical images and (D) percentage of cell growth of SAS cells transfected with siRNA (40 nM) or negative control siRNA (40 nM) for $24 \mathrm{~h}$ and cultured for $72 \mathrm{~h}$, followed by viable cell counting. Each value in D represents the percentage of cell growth compared with the vehicle (non-siRNA) control from six independent experiments. The black column represents the growth of cells transfected with FABP4-siRNA; the white column represents the growth of cells transfected with the negative control-siRNA. Error bars represent standard errors. ${ }^{* *} \mathrm{P}<0.01$ vs. negative control.

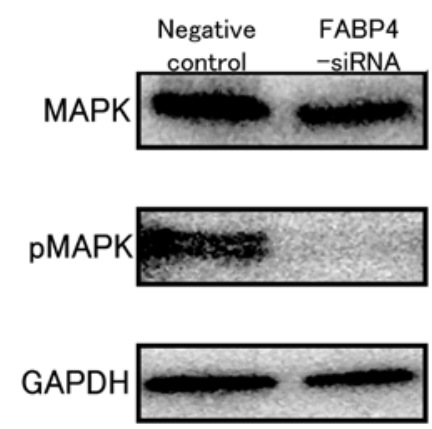

Figure 3. FABP4-specific siRNA inhibits expression of mitogen-activated protein kinase (MAPK) and its phosphorylation. A representative western blot analysis shows MAPK and p-MAPK expression in SAS cells. SAS cells were treated with $F A B P 4$-specific siRNA $(40 \mathrm{nM})$ or negative control siRNA $(40 \mathrm{nM})$. Samples were collected $12 \mathrm{~h}$ after the treatment. GAPDH was used as a standard for equivalent application.

in the non-tumor area in the same tissue samples. Therefore, FABP4 expression in tumors may affect SCC cell growth. In fact, we showed that suppression of FABP4 protein by the $F A B P 4$-specific siRNA clearly inhibited the growth of SCC cell lines. These results clearly indicate the important role of FABP4 in SCC growth.

FABPs actively facilitate the transport of lipids to specific cellular compartments, including lipid droplets for storage; endoplasmic reticulum for signaling, trafficking and membrane synthesis; mitochondria or peroxisome for oxidation; cytosolic or other enzymes for activity regulation; nuclei for lipid-mediated transcriptional regulation; or even outside the cell for autocrine or paracrine signaling. Among the FABPs,

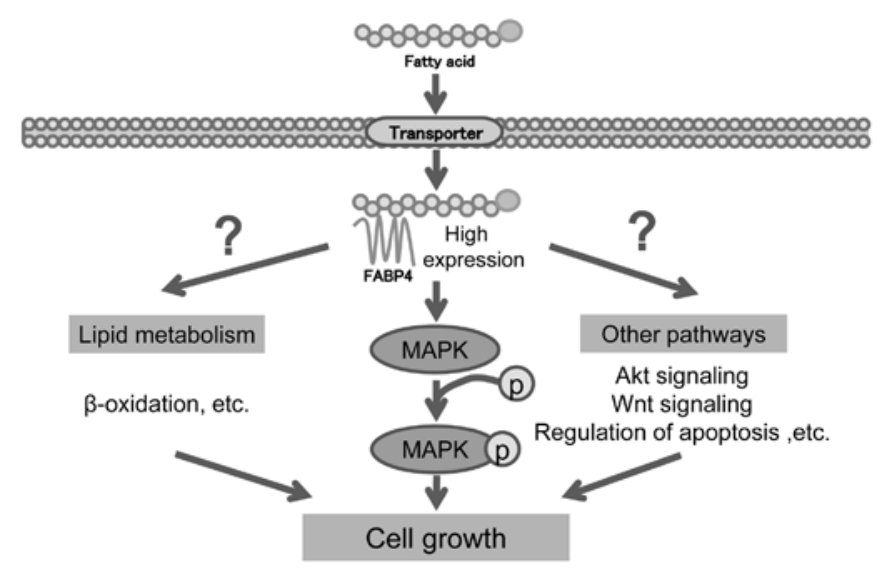

Figure 4. Schematic illustration of possible mechanisms of cell growth inhibition by FABP4-specific siRNA.

FABP4 is highly expressed in adipocytes, macrophages and dendritic cells and affects these cells in various manners (5). In cancer cells, FABP4 transports energy by carrying fatty acids, encouraging metastasis and tumor cell growth (10). Yet, FABP4 performs other roles in tumor growth, through its various functions. Therefore, we studied the role of FABP4 in the MAPK pathway and the mechanisms of growth inhibition induced by FABP4 suppression.

As the MAPK pathway helps to mediate cell proliferation and cancer growth, it has been widely studied as a potential target for cancer therapy (19-21). In fact, our present research showed decreased MAPK expression and phosphorylation $12 \mathrm{~h}$ following treatment with $F A B P 4$-specific siRNA, 
which indicates that FABP4 affects cell growth through the MAPK pathway. Notably, expression of MAPK itself was also suppressed by FABP4 knockdown; FABP4 may affect transcription of MAPK, which implies a complex role for FABP4 in tumor growth. Inhibition of the MAPK pathway may be one of the several mechanisms through which FABP4 mediates tumor growth (Fig. 4). Further investigation is warranted.

In the present study, FABP4 expression in the tumor tissues was not correlated with age, gender, histological tumor differentiation or survival rate of the cases. Of our 27 cases, 12 had neck lymph node metastasis, all 12 of whom showed FABP4 expression in lymph nodes, similar to that in the primary tumors (data not shown). Thus, FABP4 expression may affect metastasis to neck lymph nodes; this role merits further investigation.

In summary, we demonstrated FABP4 expression in human tongue SCC tissues and cultured SCC cells. Our results suggest an important role for FABP4 in SCC growth and indicate that FABP4 is a potential target for the therapy of oral SCC.

\section{Acknowledgements}

The present study was supported in part by grants (T245928310 to Y.T.) from the Japanese Society for the Promotion of Science.

\section{References}

1. Prince S and Bailey BM: Squamous carcinoma of the tongue: review. Br J Oral Maxillofac Surg 37: 164-174, 1999.

2. Okura M, Hiranuma T, Adachi T, et al: Induction chemotherapy is associated with an increase in the incidence of locoregional recurrence in patients with carcinoma of the oral cavity: results from a single institution. Cancer 82: 804-815, 1998.

3. Goepfert H: Squamous cell carcinoma of the head and neck: past progress and future promise. CA Cancer J Clin 48: 195-198, 1998.

4. Schroeder F, Petrescu AD, Huang H, et al: Role of fatty acid binding proteins and long chain fatty acids in modulating nuclear receptors and gene transcription. Lipids 43: 1-17, 2008.

5. Furuhashi $\mathrm{M}$ and Hotamisligil GS: Fatty acid-binding proteins: role in metabolic diseases and potential as drug targets. Nat Rev Drug Discov 7: 489-503, 2008.

6. Storch J and Thumser AE: The fatty acid transport function of fatty acid-binding proteins. Biochim Biophys Acta 1486: 28-44, 2000

7. Hotamisligil GS, Johnson RS, Distel RJ, Ellis R, Papaioannou VE and Spiegelman BM: Uncoupling of obesity from insulin resistance through a targeted mutation in aP2, the adipocyte fatty acid binding protein. Science 274: 1377-1379, 1996.
8. Uysal KT, Scheja L, Wiesbrock SM, Bonner-Weir S and Hotamisligil GS: Improved glucose and lipid metabolism in genetically obese mice lacking aP2. Endocrinology 141: 3388-3396, 2000

9. Makowski L, Boord JB, Maeda K, et al: Lack of macrophage fatty-acid-binding protein aP2 protects mice deficient in apolipoprotein E against atherosclerosis. Nat Med 7: 699-705, 2001.

10. Nieman KM, Kenny HA, Penicka CV, et al: Adipocytes promote ovarian cancer metastasis and provide energy for rapid tumor growth. Nat Med 17: 1498-1503, 2011.

11. Masuda T, Wada K, Nakajima A, et al: Critical role of peroxisome proliferator-activated receptor $\gamma$ on anoikis and invasion of squamous cell carcinoma. Clin Cancer Res 11: 4012-4021, 2005.

12. Nagata $M$, Wada $K$, Nakajima A, et al: Role of myeloid cell leukemia-1 in cell growth of squamous cell carcinoma. J Pharmacol Sci 110: 344-353, 2009.

13. Kusayama M, Wada K, Nagata M, et al: Critical role of aquaporin 3 on growth of human esophageal and oral squamous cell carcinoma. Cancer Sci 102: 1128-1136, 2011.

14. Ishimoto S, Wada K, Tanaka N, et al: Role of endothelin receptor signalling in squamous cell carcinoma. Int J Oncol 40: 1011-1019, 2012.

15. Ishimoto S, Wada K, Usami Y, et al: Differential expression of aquaporin 5 and aquaporin 3 in squamous cell carcinoma and adenoid cystic carcinoma. Int J Oncol 41: 67-75, 2012.

16. Tan NS, Shaw NS, Vinckenbosch N, et al: Selective cooperation between fatty acid binding proteins and peroxisome proliferatoractivated receptors in regulating transcription. Mol Cell Biol 22: 5114-5127, 2002

17. Ishida H, Wada K, Masuda $\mathrm{T}$, et al: Critical role of estrogen receptor on anoikis and invasion of squamous cell carcinoma. Cancer Sci 98: 636-643, 2007.

18. Takahashi H, Fujita K, Fujisawa T, et al: Inhibition of peroxisome proliferator-activated receptor gamma activity in esophageal carcinoma cells results in a drastic decrease of invasive properties. Cancer Sci 97: 854-860, 2006.

19. Roux PP and Blenis J: ERK and p38 MAPK-activated protein kinases: a family of protein kinases with diverse biological functions. Microbiol Mol Biol Rev 68: 320-344, 2004.

20. Roberts PJ and Der CJ: Targeting the Raf-MEK-ERK mitogenactivated protein kinase cascade for the treatment of cancer. Oncogene 26: 3291-3310, 2007.

21. Santarpia L, Lippman SM and El-Naggar AK: Targeting the MAPK-RAS-RAF signaling pathway in cancer therapy. Expert Opin Ther Targets 16: 103-119, 2012.

22. Boiteux G, Lascombe I, Roche E, et al: A-FABP, a candidate progression marker of human transitional cell carcinoma of the bladder, is differentially regulated by PPAR in urothelial cancer cells. Int J Cancer 124: 1820-1828, 2009.

23. Uma RS, Naresh KN, D'Cruz AK, Mulherkar R and Borges AM: Metastasis of squamous cell carcinoma of the oral tongue is associated with down-regulation of epidermal fatty acid binding protein (E-FABP). Oral Oncol 43: 27-32, 2007.

24. Fang LY, Wong TY, Chiang WF and Chen YL: Fatty-acidbinding protein 5 promotes cell proliferation and invasion in oral squamous cell carcinoma. J Oral Pathol Med 39: 342-348, 2010. 